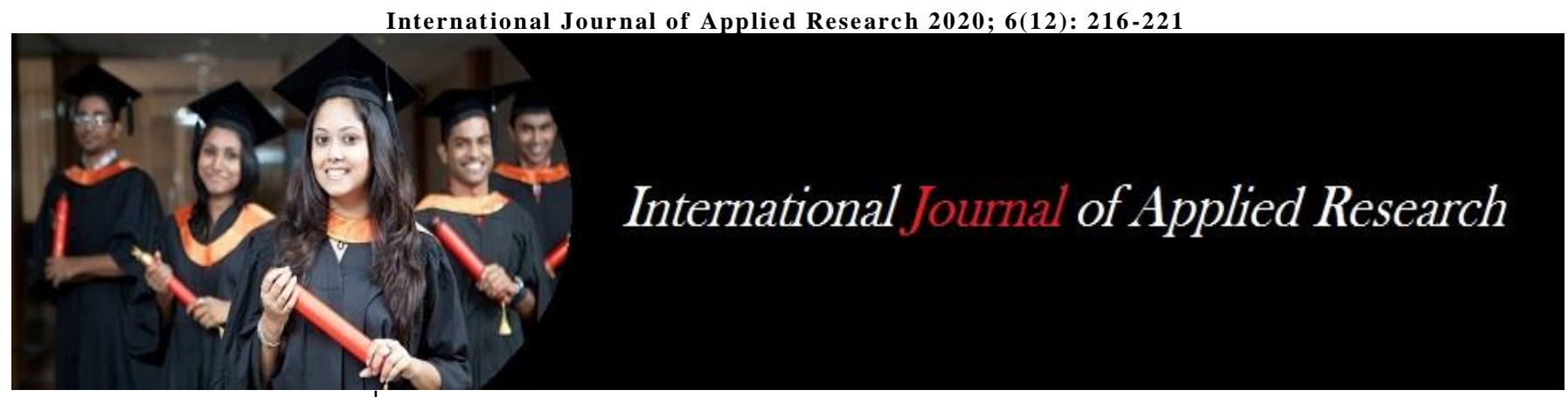

ISSN Print: 2394-7500

ISSN Online: 2394-5869

Impact Factor: 8.4

IJAR 2020; 6(12): 216-221

www.allresearchjournal.com

Received: 08-10-2020

Accepted: 10-11-2020

Shalini Singh

Department of Chemistry,

Uttarakhand Open University

Vishwavidhyalaya Marg,

Haldwani (Nainital),

Uttarakhand, India

\section{Correlation studies among soil properties, climatic conditions and essential oil constituents of Origanum vulgare L. from Central Himalaya, India}

\section{Shalini Singh}

DOI: https://doi.org/10.22271/allresearch.2020.v6.i12d.8086

\begin{abstract}
Environmental conditions and soil properties plays important role to regulate the plant metabolism. Considering the importance of essential oil of Origanum vulgare L., this study was done to understand the oil profile with microclimatic conditions and soil properties in wild conditions. The plant materials were collected from ten sites of Uttarakhand, India with their soil samples. Correlation was established among essential oil major constituents, micro and macronutrients, microclimatic conditions, physical properties of soil by using Microsoft Excel XP. Available nitrogen in soil was positively correlated with linalool, bornyl acetate, $(E)$-caryophyllene, germacrene D and $\beta$-bisabolene while negatively correlated with thymol. Thymol and caryophyllene oxide were found to be positively correlated with available potassium. Available $\mathrm{Zn}$ was positively correlated with $\beta$-bisabolene. Total iron $(\mathrm{Fe})$ present in the soil was found to be positively correlated with total p-cymene and $\gamma$-terpinene in the oils. $\mathrm{pH}$ of soil was found to be negatively correlated with thymol and positively correlated with elemol. Percent organic carbon $(\mathrm{OC} \%)$ was negatively correlated with p-cymene, $(E)$-ocimene and caryophyllene oxide. In our study water holding capacity is positively correlated with caryophyllene oxide. The results of present study indicate the significant role of macro and micronutrients and other soil properties on the essential oil composition.
\end{abstract}

Keywords: Essential oil, microclimatic conditions, macronutrients, micronutrients, Origanum vulgare L.

\section{Introduction}

Origanum vulgare L. (family Labiateae; Lamiaceae), is a multipurpose medicinal plant commonly known as Bantulsi, oregano or Himalayan marjoram in India ${ }^{[1]}$. It is widely distributed in the mild and temperate climate of Eurasia, North Africa and America from 7000 to $12,000 \mathrm{ft} .{ }^{[2-3]}$. It is $20-80 \mathrm{~cm}$ high, erect, perennial and aromatic herb with ovate, entire, stalked leaves $1-4 \mathrm{~cm}$ and small pale pink flowers ${ }^{[4]}$. Origanum vulgare L. is the only species of the genus Origanum which is found in India ${ }^{[3]}$. Origanum is used as herbal tea spices salads, sausages olives in meat by local people. Since ancient times, $O$. vulgare L. has been used as stimulant, stomachic, analgesic, expectorant, sedative, antiparasitic, carminative and diaphoretic ${ }^{[5-6]}$. A number of reports have been published on the antimicrobial, antifungal, antiviral, analgesic, antioxidant and anti-inflammatory activities of Origanum vulgare L. ${ }^{[7-13]}$. The essential oil of $O$. vulgare has been widely studied from many countries [14-16]

The most important biologically active compounds reported to be present in $O$. vulgare are carvacrol and thymol followed by $\gamma$-terpinene, $p$-cymene, linalool and terpinen-4-ol. Among these, $\gamma$-terpinene and p-cymene are supposed to be the biological precursors of the phenolic monoterpenes ${ }^{[1]}$. Four chemotypes carvacrol, (Z)- $\alpha$-bisabolene, linalyl acetate and caryophyllene oxide/germacrene $\mathrm{D} /(\mathrm{E})-\beta$-caryophyllene were investigated from Iranian Origanum vulgare L. grown under similar conditions ${ }^{[17]}$. De Martino et al. in 2009 reported three chemotypes of $O$. vulgare L. growing wild in Campania (Southern Italy) which were rich in carvacrol/thymol, thymol/ $\alpha$-terpineol and linalyl acetate/linalool ${ }^{[18]}$. The essential oil composition of aromatic plants depends upon various environmental factors like nature of soil, climatic conditions, altitude, temperature, moisture and harvesting conditions ${ }^{[19-20]}$. The content of macro and micronutrients in the soil as well as in the plants are the one of the
Corresponding Author: Shalini Singh Department of Chemistry, Uttarakhand Open University Vishwavidhyalaya Marg, Haldwani (Nainital), Uttarakhand, India 
important factors, which affects the essential oil composition of aromatic and medicinal plants. They play a very important role in the biogenetic pathways of different secondary metabolites of the oil. Kanias et al. (1998) [21] reported that iron, chromium, and scandium showed a negative significant correlation with carvacrol and positive one with thymol. Application of phosphorus in nutrient solution was found to increases the percentage of p-cymene accompanied by a decrease in the percentage of carvacrol in the case of leaves of $O$. dictamnus ${ }^{[22]}$. Effect of nitrogen fertilization on the essential oil of $O$. vulgare L. has been reported by Said-Al Ahl et al. (2009) ${ }^{[23]}$.

To the best our knowledge no work has been reported on the effect of soil and geographic conditions on the essential oil composition of the wild $O$. vulgare L. in Uttarakhand. Therefore, the aim of present study is to explore the effect of soil and microclimatic conditions on this important genus.

\section{Materials and methods}

\section{Plant material and soil samples}

Collection of plant material (at full blooming stage) along with its soil samples $(0-20 \mathrm{~cm})$ were done from ten sites of Kumaun Himalayan region. The plants specimens were authenticated at Botany Department, Kumaun University, Nainital (Voucher no.-2036). The geographical and climatic conditions are given in Table 1.

\section{Determination of physicochemical properties of soil}

Soil texture was determined by using International hydrometer method using sodium meta phosphate $(5 \%)$ as dispersing agent (Kilmer and Alexander, 1949) [24]. Measurement of the soil $\mathrm{pH}$ and electrical conductivity (EC) of the soil suspension (1:2 soil and water ratio) was done by using $\mathrm{pH}$ meter and EC meter (Jackson, 1958) ${ }^{[25]}$. Soil organic-carbon (\%OC) content was determined by using Walkley and Black method described by Jackson (1958) ${ }^{[25]}$ Cation exchange capacity (CEC) was determined by the $\mathrm{NH}^{+}{ }^{+}$saturation method given by Chapman. Water holding capacity (WHC) of the soils was determined by using Hilgard apparatus (Black, 1965) ${ }^{[26]}$. Total nitrogen (N), available phosphorus $\left(\mathrm{P}_{2} \mathrm{O}_{5}\right)$ and available potassium $(\mathrm{K})$ were determined by using Kjeldahl method, Olsen's method and flame photometer respectively.

\section{Soil sample preparation for micronutrient analysis.}

Sample for the analysis of total metal content (Piper, 1942) [27] in soil were prepared by digestion with $\mathrm{HClO}_{4}-\mathrm{HF}$ method. $1.0 \mathrm{~g}$ of soil wetted with $5 \mathrm{ml}$ distilled water. $2 \mathrm{ml}$ of $\mathrm{HClO}_{4}$ and $12 \mathrm{~mL}$ of $\mathrm{HF}$ added and heated till dryness. Now $2 \mathrm{~mL}$ of $\mathrm{HClO}_{4}$ and $5 \mathrm{~mL}$ of distilled water were added and heated to dryness residue was dissolved in $8 \mathrm{~mL}$ of $\mathrm{HCl}$ and $20 \mathrm{~mL}$ of water and distilled water was added to make up the volume $50 \mathrm{~mL}$.

The procedure developed by Lindsay and Norvell (1978) ${ }^{[28]}$ was followed to prepare soil samples for available metal content. $20 \mathrm{~mL}$ extracting solution $(0.005 \mathrm{M}$ DTPA $+0.1 \mathrm{M}$ triethanolamine $+0.01 \mathrm{M} \mathrm{CaCl}_{2}$ ) was added to $10 \mathrm{~g}$ soil taken in a conical flask. Suspention in flasks was shaken for two hours at a speed of 120 cycles per minute on a horizontal shaker. After shaking, the suspension was filtered through a Whatman no. 42 filter paper.

\section{Heavy metal analysis in soil samples}

Atomic absorption spectrophotometer (GBC-902 and Avanta sigma Models) was used to analyze the $\mathrm{Zn}, \mathrm{Cu}, \mathrm{Mn}$, and $\mathrm{Fe}$.

\section{Statistical analysis}

Experimental data were processed using Microsoft Excel XP. Correlation coefficients were calculated among oil major constituents, micro and macronutrients, microclimatic conditions and physical properties of soil. Significance level of correlation coefficient was checked on probability level of $\mathrm{p}<0.05$ and $\mathrm{p}<0.01$.

\section{Results and discussion}

\section{Physicochemical parameters of the soil samples}

The soil samples were analysed for their physicochemical properties (Table 2). The analysis was done in triplicate and represented as the mean value \pm standard deviation $( \pm \mathrm{SD})$. The soils were loamy sand and sandy loam. They were acidic to neutral in nature ( $\mathrm{pH} 5.31$ to 7.41). The EC, OC \%, CEC and WHC values of most of the soil samples were observed to be within the limits. Furthermore, macro and micronutrients content also falls within the permissible limits.

\section{Essential oil components}

The major components in the essential oils of the $O$. vulgare L. collected from ten sites have already been reported in our previous studies (Pande et al., 2012; Singh et al., 2013) (29-30] $^{\text {29 }}$ Mukteshwer, Rushi village and Kilbury, Nanital, Mussoorie collections were found to be rich in linalool $(5.1 \%-9.7 \%)$, germacrene $\mathrm{D}(6.3 \%-18.00 \%),(E)$-caryophyllene $(9.2 \%$ $16 \%)$ and bornyl acetate (12.6-18.6\%). Thymol (5.1\%), germacrene D (5.7\%). carvacrol (7.5\%), $\alpha$-cadinol $(9.3 \%),(\mathrm{E})$ - caryophyllene $(10.4 \%)$ and linalool(10.9\%) were the major components obtained from the oil of Ramgarh. The oil collected from Dhoulchina and Champawat were rich in p-cymene (6.7-9.8\%), $\gamma$-terpinene (12.4-14.0\%), carvacrol (12.4\%-20.9\%) and thymol (29.7$35.1 \%)$. The composition of oil from Dharchula and Munsiyari showed the remarkable presence of thymol (30.2$55.1 \%)$ followed by aliphatic hydrocarbons (12.8-35.1\%) and caryophyllene oxide (7.5-7.6\%).

\section{Correlation of soil properties with essential oil components}

Simple correlation coefficients $\left(\mathrm{r}^{2}\right)$ shown in Table 3 the soil $\mathrm{pH}$ was found to be negatively correlated with thymol $\left(\mathrm{r}^{2}=-\right.$ $0.671, \mathrm{P}<0.05)$ and positively correlated with elemole $\left(\mathrm{r}^{2}=-\right.$ $0.647, \mathrm{P}<0.05)$. Percent organic carbon represent negative correlation with $\mathrm{p}$-cymene $\left(\mathrm{r}^{2}=-0.759, \mathrm{P}<0.05\right), \gamma$-terpinene $\left(r^{2}=-0.692, P<0.05\right)$ and caryophyllene oxide $\left(r^{2}=-0.698\right.$, $\mathrm{P}<0.05)$. The study done by Dunford Vazquez (2005) ${ }^{[31]}$ to correlate the effect of moisture on Maxican oregano showed that amount of water received by the plant did not have any significant effect on thymol and carvacrol of the oil. In our study, water holding capacity was positively correlated with caryophyllene oxide $\left(\mathrm{r}^{2}=0.633, \mathrm{P}<0.05\right)$.

\section{Correlation of macronutrients with essential oil components}

According to simple correlation coefficients $\left(r^{2}\right)$ shown in Table 4 the available nitrogen was positively correlated with linalool $\left(\mathrm{r}^{2}=0.672, \quad \mathrm{P}<0.05\right)$, bornyl acetate $\left(\mathrm{r}^{2}=0.642\right.$, $\mathrm{P}<0.05)$, (E)-caryophyllene $\left(\mathrm{r}^{2}=0.684, \mathrm{P}<0.05\right)$, germacrene $\mathrm{D}\left(\mathrm{r}^{2}=0.674, \mathrm{P}<0.05\right)$ and $\beta$-bisabolene $\left(\mathrm{r}^{2}=0.649, \mathrm{P}<0.05\right)$ while negatively correlated with thymol $\left(r^{2}=-0.842\right.$, $\mathrm{P}<0.01)$. Arabaci et al. (2007) ${ }^{[32]}$ reported that nitrogen fertilizer increased the linalool content in the essential oil of 
Lavandula hybrid which also support our result in natural conditions. Omer (1999) [33] reported that nitrogen fertilization increased the biosynthesis of thymol and carvacrol while in our result, natural nitrogen concentration was negatively correlated with thymol. Available potassium was found to be positively correlated with thymol $\left(\mathrm{r}^{2}=0.709\right.$, $\mathrm{P}<0.05)$ and caryophyllene oxide $\left(\mathrm{r}^{2}=0.642, \mathrm{P}<0.05\right)$ (Table 4).

\section{Correlation of micronutrients with essential oil components}

Available $\mathrm{Zn}$ in soil was positively correlated with $\beta$ bisabolene $\left(\mathrm{r}^{2}=0.644, \mathrm{P}<0.05\right)$. (Table 5). Kanias, et al. (1998) [21] reported that chromium, iron and zinc are responsible for variation of the concentration of thymol, carvacrol and $\delta$-cadinene. As zinc participates in photosynthesis and saccharide metabolism, and as $\mathrm{CO}_{2}$ and glucose is the most likely sources of carbon utilized in terpene biosynthesis, the role of zinc becomes very important in the terpenoid biosynthesis ${ }^{[34]}$. Total iron (Fe) present in the soil was found to be positively correlated with total p-cymene $\left(\mathrm{r}^{2}=0.693, \quad \mathrm{P}<0.05\right)$ and $\gamma$-terpinene $\left(\mathrm{r}^{2}=0.736, \mathrm{P}<0.05\right)$. (Table 6). Kanias et al. $(1998)^{[21]}$ found positive correlation between iron and carvacrol in $O$. vulgare collected from Greece. Available Iron is positively correlated with germacrene $\mathrm{D}(\mathrm{r}=0.636, \mathrm{P} \leq 0.05)$ and elemol $(\mathrm{r}=0.759, \mathrm{P} \leq 0.05)$ (Table 7). Iron plays a very important role in plant metabolism ${ }^{[35]}$. It activates catalase enzymes associated with superoxide dismutase, photorespiration and the glycolate pathway. Role of micronutrients, altitude on essential oil composition has been studied by Singh et al. (2013) In this study essential oil of Craniotome furcata altitude seems one of the important factors influencing the percent variation of germacrene $D$ [36]. Under warm conditions the percentage of $(E)$-caryophyllene found more in essential oil composition of Ocimum ammericanum ${ }^{\text {[37]. In }}$ present study, no significant correlations were found between concentration of $\mathrm{Mn}$ (Table 7), $\mathrm{Cu}$ (Table 8), altitude of site and temperature (Table 9) with essential oil components.

Table 1: Geographic and climatic conditions of collection sites

\begin{tabular}{|c|c|c|c|c|c|c|c|c|c|c|}
\hline Districts & Dehradun & \multicolumn{4}{|c|}{ Nainital } & \multicolumn{2}{|c|}{ Pithoragarh } & Almora & Champawat \\
\hline $\begin{array}{c}\text { Geographic and } \\
\text { climatic conditions }\end{array}$ & $\begin{array}{c}\text { Mussoori } \\
\mathbf{e}\end{array}$ & $\begin{array}{c}\text { Mukteshwa } \\
\mathbf{r}\end{array}$ & Kilbury & Nainital & $\begin{array}{c}\text { Ramgar } \\
\mathbf{h}\end{array}$ & Rushi & Munsiyari & Dharchula & Dhoulchina & Champawat \\
\hline Altitude $(\mathrm{m})$ & 2333 & 2286 & 2134 & 2100 & 1789 & 1600 & 2235 & 2183 & 1800 & 1650 \\
\hline Temperature $\left({ }^{\circ} \mathrm{C}\right)$ & 20 & 18 & 23 & 22 & 23 & 28 & 25 & 22 & 24 & 26 \\
\hline Latitude/longitude & $30^{\circ} 27^{\prime} \mathrm{N}:$ & $29^{\circ} 28^{\prime} \mathrm{N}:$ & $29^{\circ} 23^{\prime} \mathrm{N}:$ & $29^{\circ} 23^{\prime} \mathrm{N}:$ & $29^{\circ} 23^{\prime} \mathrm{N}:$ & $29^{\circ} 23^{\prime} \mathrm{N}:$ & $30^{\circ} 04^{\prime} 37^{\prime \prime} \mathrm{N}:$ & $29^{\circ} 51^{\prime} 00^{\prime \prime} \mathrm{N}:$ & $29^{\circ} 37^{\prime} \mathrm{N}: 79^{\circ} 4$ \\
$7^{\prime} \mathrm{E}$ & $29^{\circ} 36^{\prime} \mathrm{N}: 79^{\circ}$ \\
$38^{\circ} 06^{\prime} \mathrm{E}$ & $79^{\circ} 39^{\prime} \mathrm{E}$ & $79^{\circ} 30^{\prime} \mathrm{E}$ & $79^{\circ} 30^{\prime} \mathrm{E}$ & $79^{\circ} 30^{\prime} \mathrm{E}$ & $79^{\circ} 30^{\prime} \mathrm{E}$ & $80^{\circ} 23^{\prime} 04^{\prime \prime} \mathrm{E}$ & $80^{\circ} 31^{\prime} 60^{\prime \prime} \mathrm{E}$ & 0 \\
\hline Sun/Shady side & Sunny & Shady & Sunny & Sunny & Shady & Shady & Sunny & Sunny & Shady & Sunny \\
\hline
\end{tabular}

Table 2: Physicochemical properties of collected soil samples

\begin{tabular}{|c|c|c|c|c|c|c|c|c|c|c|}
\hline \multirow{2}{*}{$\begin{array}{c}\text { Districts } \\
\text { Soil Properties }\end{array}$} & \multirow{2}{*}{\begin{tabular}{|l|} 
Dehradun \\
Mussoorie \\
\end{tabular}} & \multicolumn{5}{|c|}{ Nainital } & \multicolumn{2}{|c|}{ Pithoragarh } & \multirow{2}{*}{\begin{tabular}{|c|} 
Almora \\
Dhoulchina
\end{tabular}} & \multirow{2}{*}{\begin{tabular}{|l|} 
Champawat \\
Champawat
\end{tabular}} \\
\hline & & $\begin{array}{c}\text { Mukteshw } \\
\text { er }\end{array}$ & Kilbury & Nainital & Ramgarh & Rushi & Munsiyari & Dharchula & & \\
\hline Soil Texture & $\begin{array}{l}\text { Sandy } \\
\text { loam }\end{array}$ & $\begin{array}{c}\text { Loamy } \\
\text { sand }\end{array}$ & $\begin{array}{l}\text { Loamy } \\
\text { sand }\end{array}$ & $\begin{array}{l}\text { Loamy } \\
\text { sand }\end{array}$ & $\begin{array}{l}\text { Sandy } \\
\text { loam }\end{array}$ & $\begin{array}{l}\text { Loamy } \\
\text { sand }\end{array}$ & $\begin{array}{l}\text { Sandy } \\
\text { loam }\end{array}$ & $\begin{array}{c}\text { Sandy } \\
\text { loam }\end{array}$ & Sandy loam & Loamy sand \\
\hline Soil pH $(1: 2)$ & $7.40 \pm 0.11$ & $7.41 \pm 0.10$ & $7.32 \pm 0.11$ & $5.60 \pm 0.61$ & $6.62 \pm 1.00$ & $6.22 \pm 0.61$ & $5.41 \pm 0.20$ & $5.31 \pm 0.40$ & $5.81 \pm 0.20$ & $6.30 \pm 0.30$ \\
\hline Organic carbon $(\%)$ & $3.00 \pm 0.70$ & $2.65 \pm 0.35$ & $1.21 \pm 0.30$ & $3.23 \pm 0.07$ & $3.15 \pm 0.04$ & $3.90 \pm 0.61$ & $4.20 \pm 0.40$ & $4.10 \pm 0.71$ & $1.70 \pm 0.40$ & $0.60 \pm 0.30$ \\
\hline Electrical conductivity $\left(\mathrm{dS} \mathrm{cm}^{-1}\right)$ & $0.18 \pm 0.01$ & $0.34 \pm 0.02$ & $0.10 \pm 0.00$ & $0.07 \pm 0.00$ & $0.19 \pm 0.01$ & $0.10 \pm 0.00$ & $0.11 \pm 0.02$ & $0.20 \pm 0.00$ & $0.12 \pm 0.02$ & 0.05 \\
\hline $\begin{array}{l}\text { Cation exchange capacity (c mol } \\
\qquad \mathrm{kg}^{-1} \text { ) }\end{array}$ & $18.11 \pm 0.21$ & $38.11 \pm 0.30$ & $19.72 \pm 0.20$ & $25.83 \pm 0.51$ & $15.12 \pm 1.38$ & $16.31 \pm 1.21$ & $27.30 \pm 2.01$ & $10.30 \pm 0.20$ & $10.31 \pm 0.29$ & $27.36 \pm 0.79$ \\
\hline Water holding capacity (\%) & $58.31 \pm 0.60$ & $46.10 \pm 1.50$ & $49.71 \pm 2.19$ & $39.61 \pm 1.56$ & $35.10 \pm 0.19$ & $38.21 \pm 1.65$ & $54.59 \pm 1.68$ & $56.00 \pm 2.50$ & $37.87 \pm 2.26$ & $39.69 \pm 0.47$ \\
\hline Available nitrogen $(\mathrm{kg} / \mathrm{hc})$ & $0.012 \pm 0.29$ & $\begin{array}{c}0.0138 \pm 0.0 \\
3\end{array}$ & $0.011 \pm 0.09$ & $0.009 \pm 0.25$ & $0.012 \pm 1.54$ & $0.012 \pm 0.67$ & $0.008 \pm 0.50$ & $0.004 \pm 0.01$ & $0.005 \pm 1.34$ & $0.009 \pm 0.78$ \\
\hline Total nitrogen $(\mathrm{kg} / \mathrm{hc})$ & $0.250 \pm 0.01$ & $0.350 \pm 0.03$ & $0.210 \pm 0.01$ & $0.180 \pm 0.02$ & $0.300 \pm 0.03$ & $0.240 \pm 0.05$ & $0.280 \pm 0.06$ & $0.300 \pm 0.05$ & $0.200 \pm 0.00$ & $0.280 \pm 0.01$ \\
\hline Available phosphorus $(\mathrm{kg} / \mathrm{hc})$ & $\begin{array}{c}0.0009 \pm 0.0 \\
0\end{array}$ & $\begin{array}{c}0.0024 \pm 0.0 \\
0\end{array}$ & $\begin{array}{c}0.0037 \pm 0.0 \\
01\end{array}$ & $\begin{array}{c}0.0014 \pm 0.0 \\
0\end{array}$ & $\begin{array}{c}0.0033 \pm 0.0 \\
0\end{array}$ & $\begin{array}{c}0.0007 \pm 0.0 \\
0\end{array}$ & $\begin{array}{c}0.0011 \pm 0.0 \\
0\end{array}$ & \begin{tabular}{|c|}
$0.0030 \pm 0.0$ \\
0
\end{tabular} & $0.0026 \pm 0.00$ & $0.0009 \pm 0.00$ \\
\hline Available potassium $(\mathrm{kg} / \mathrm{hc})$ & $0.02 \pm 0.001$ & $\begin{array}{c}0.010 \pm 0.00 \\
1\end{array}$ & $0.03 \pm 0.010$ & $0.01 \pm 0.001$ & $\begin{array}{c}0.020 \pm 0.01 \\
0\end{array}$ & $0.00 \pm 0.002$ & $0.02 \pm 0.010$ & $0.24 \pm 0.030$ & $0.02 \pm 0.020$ & $0.020 \pm 0.001$ \\
\hline Total $\mathrm{Zn}$ content $\left(\mathrm{mg} \mathrm{kg}^{-1}\right)$ & \begin{tabular}{|c|}
$54.280 \pm 0.5$ \\
7
\end{tabular} & $\begin{array}{c}91.680 \pm 0.4 \\
3\end{array}$ & \begin{tabular}{|c|}
$45.280 \pm 1.4$ \\
2
\end{tabular} & \begin{tabular}{|c|}
$40.500 \pm 2.1$ \\
3
\end{tabular} & $\begin{array}{c}35.280 \pm 0.6 \\
7\end{array}$ & \begin{tabular}{|c|}
$26.880 \pm 1.1$ \\
7
\end{tabular} & $\begin{array}{c}38.670 \pm 0.0 \\
8\end{array}$ & \begin{tabular}{|c|}
$35.050 \pm 0.9$ \\
1
\end{tabular} & $32.150 \pm 0.48$ & $43.620 \pm 0.63$ \\
\hline Total Fe content $\left(\mathrm{mg} \mathrm{kg}^{-1}\right)$ & $\begin{array}{c}522.060 \pm 1 \\
79 \\
\end{array}$ & \begin{tabular}{|c|}
$559.250 \pm 2$. \\
94
\end{tabular} & $\begin{array}{c}528.870 \pm 6 \\
99\end{array}$ & \begin{tabular}{|c|}
$536.480 \pm 0$ \\
49
\end{tabular} & \begin{tabular}{|c|}
$526.470 \pm 8$ \\
85
\end{tabular} & \begin{tabular}{|c|}
$516.860 \pm 4$. \\
09
\end{tabular} & \begin{tabular}{|c|}
$519.330 \pm 1$ \\
03
\end{tabular} & \begin{tabular}{|c|}
$525.630 \pm 5$ \\
03
\end{tabular} & $\begin{array}{c}543.640 \pm 14 \\
00\end{array}$ & $\begin{array}{c}564.450 \pm 12 \\
87\end{array}$ \\
\hline Total Mn content $\left(\mathrm{mg} \mathrm{kg}^{-1}\right)$ & $\begin{array}{c}220.900 \pm 0 \\
59\end{array}$ & $\begin{array}{c}348.020 \pm 1 \\
42\end{array}$ & \begin{tabular}{|c|}
$252.010 \pm 0$. \\
62
\end{tabular} & \begin{tabular}{|c|}
$264.400 \pm 0$ \\
59
\end{tabular} & $\begin{array}{c}219.350 \pm 0 \\
04\end{array}$ & \begin{tabular}{|c|}
$84.150 \pm 1.8$ \\
2
\end{tabular} & \begin{tabular}{|c|}
$165.000 \pm 0$ \\
85
\end{tabular} & $\begin{array}{c}160.100 \pm 1 \\
63\end{array}$ & $\begin{array}{c}194.620 \pm 0.5 \\
0\end{array}$ & \begin{tabular}{|c|}
$185.810 \pm 2.1$ \\
8
\end{tabular} \\
\hline Total $\mathrm{Cu}$ content $\left(\mathrm{mg} \mathrm{kg}^{-1}\right)$ & \begin{tabular}{|c|}
$19.830 \pm 0.8$ \\
8 \\
\end{tabular} & \begin{tabular}{|c|}
$15.550 \pm 0.0$ \\
2 \\
\end{tabular} & \begin{tabular}{|c|}
$34.680 \pm 0.2$ \\
7
\end{tabular} & $\begin{array}{c}15.300 \pm 0.5 \\
9\end{array}$ & $\begin{array}{c}10.830 \pm 0.8 \\
8\end{array}$ & $\begin{array}{c}10.700 \pm 1.7 \\
5\end{array}$ & \begin{tabular}{|c|}
$24.630 \pm 0.6$ \\
8
\end{tabular} & $\begin{array}{c}23.400 \pm 0.3 \\
5\end{array}$ & $15.500 \pm 1.15$ & $12.900 \pm 0.71$ \\
\hline Available Zn content $\left(\mathrm{mg} \mathrm{kg}^{-1}\right)$ & $4.980 \pm 2.35$ & $\begin{array}{c}10.260 \pm 0.6 \\
7\end{array}$ & $9.450 \pm 1.18$ & $7.370 \pm 0.99$ & $0.800 \pm 0.40$ & $0.650 \pm 0.24$ & $0.950 \pm 0.32$ & $0.780 \pm 0.05$ & $0.610 \pm 0.32$ & $1.240 \pm 0.28$ \\
\hline Available Fe content $\left(\mathrm{mg} \mathrm{kg}^{-1}\right)$ & $\begin{array}{c}91.700 \pm 1.3 \\
9\end{array}$ & $\begin{array}{c}57.980 \pm 0.3 \\
7\end{array}$ & $\begin{array}{c}29.940 \pm 0.8 \\
1\end{array}$ & \begin{tabular}{|c|}
$28.770 \pm 2.4$ \\
2
\end{tabular} & $\begin{array}{c}33.280 \pm 0.7 \\
1\end{array}$ & $\begin{array}{c}29.000 \pm 0.0 \\
4\end{array}$ & $\begin{array}{c}30.000 \pm 0.9 \\
5\end{array}$ & $\begin{array}{c}36.680 \pm 2.3 \\
5\end{array}$ & $22.650 \pm 1.98$ & $35.380 \pm 0.55$ \\
\hline Available Mn content $\left(\mathrm{mg} \mathrm{kg}^{-1}\right)$ & \begin{tabular}{|c|}
$17.110 \pm 0.4$ \\
2 \\
\end{tabular} & \begin{tabular}{|c|}
$17.400 \pm 0.0$ \\
8 \\
\end{tabular} & $\begin{array}{c}14.560 \pm 0.5 \\
1\end{array}$ & $\begin{array}{c}15.430 \pm 0.4 \\
2 \\
\end{array}$ & $\begin{array}{c}10.000 \pm 0.1 \\
1\end{array}$ & $3.000 \pm 0.91$ & $6.200 \pm 0.63$ & \begin{tabular}{|c|}
$14.280 \pm 0.4$ \\
8
\end{tabular} & $7.080 \pm 0.43$ & $6.680 \pm 0.23$ \\
\hline Available Cu content $\left(\mathrm{mg} \mathrm{kg}^{-1}\right)$ & $1.820 \pm 0.01$ & $7.330 \pm 0.25$ & $2.260 \pm 0.01$ & $1.830 \pm 0.60$ & $0.400 \pm 0.28$ & \begin{tabular}{|c|}
$00.320 \pm 0.0$ \\
2
\end{tabular} & $0.900 \pm 0.06$ & $1.120 \pm 0.01$ & $0.150 \pm 0.09$ & $0.530 \pm 0.50$ \\
\hline
\end{tabular}


Table 3. Correlation matrix $\left(\mathrm{r}^{2}\right)$ between physical properties of soil and major constituents of essential oil

\begin{tabular}{|c|c|c|c|c|c|c|c|c|c|c|c|c|c|c|c|c|c|}
\hline 1 & 2 & 3 & 4 & 5 & 6 & 7 & 8 & 9 & 10 & 11 & 12 & 13 & 14 & 15 & 16 & 17 & 18 \\
\hline $\mathrm{pH}$ & EC & $\begin{array}{c}\text { OC } \\
\%\end{array}$ & CEC & $\begin{array}{c}\text { Mois } \\
\text { ture } \\
\text { conte } \\
\text { nt }\end{array}$ & $\begin{array}{c}\text { p- } \\
\text { Cymen } \\
e\end{array}$ & $\begin{array}{c}r- \\
\text { Terpinen } \\
\text { e }\end{array}$ & $\begin{array}{c}\text { Linalo } \\
\text { ol }\end{array}$ & $\begin{array}{c}\text { Borny } \\
\text { l } \\
\text { acetat } \\
\text { e }\end{array}$ & $\begin{array}{c}\text { Thym } \\
\text { ol }\end{array}$ & $\begin{array}{c}\text { Carvacr } \\
\text { ol }\end{array}$ & \begin{tabular}{|c|} 
(E)- \\
Caryo \\
phyllen \\
e
\end{tabular} & $\begin{array}{c}\text { Germacre } \\
\text { ne D }\end{array}$ & $\begin{array}{c}\text { Bicycle } \\
\text { germacre } \\
\text { ne }\end{array}$ & \begin{tabular}{|c|}
$\beta-$ \\
Bisabolen \\
$\mathbf{e}$
\end{tabular} & $\begin{array}{c}\text { Elem } \\
\text { ol }\end{array}$ & $\begin{array}{c}\text { Caryophylle } \\
\text { ne oxide }\end{array}$ & $\begin{array}{c}\text { Aliphatic } \\
\text { hydrocarbo } \\
\text { ns }\end{array}$ \\
\hline $\begin{array}{c}1.0 \\
0 \\
\end{array}$ & $\begin{array}{c}0.39 \\
8\end{array}$ & $\begin{array}{c}- \\
0.422 \\
\end{array}$ & 0.311 & 0.094 & -0.100 & -0.116 & 0.544 & 0.509 & $-0.671^{*}$ & -0.233 & 0.470 & 0.578 & 0.045 & 0.521 & $0.647^{*}$ & -0.541 & -0.562 \\
\hline & 1.00 & 0.406 & 0.137 & 0.305 & -0.611 & -0.524 & 0.218 & 0.259 & -0.241 & -0.377 & 0.177 & 0.391 & 0.167 & 0.283 & 0.472 & 0.254 & -0.058 \\
\hline & & 1.00 & -0.151 & 0.299 & $-0.759^{*}$ & $-0.692^{*}$ & -0.097 & 0.048 & 0.047 & -0.527 & 0.023 & 0.190 & 0.212 & 0.108 & 0.159 & $0.698^{*}$ & 0.542 \\
\hline & & & & 1.00 & \begin{tabular}{|l|}
-0.392 \\
\end{tabular} & -0.546 & -0.482 & -0.070 & 0.233 & -0.508 & -0.308 & 0.067 & -0.283 & 0.025 & 0.259 & $0.633^{*}$ & 0.528 \\
\hline & & & & & 0.151 & 0.130 & 0.406 & 0.614 & -0.446 & -0.279 & 0.541 & 0.184 & 0.010 & 0.499 & 0.135 & -0.449 & -0.369 \\
\hline & & & & & -0.209 & -0.304 & -0.440 & -0.508 & 0.325 & 0.033 & -0.575 & -0.100 & -0.228 & -0.412 & 0.085 & 0.495 & 0.558 \\
\hline & & & & & -0.035 & 0.037 & -0.393 & -0.510 & 0.586 & 0.392 & -0.480 & -0.372 & -0.066 & -0.483 & -0.369 & 0.441 & 0.269 \\
\hline
\end{tabular}

* Correlation is significant at the 0.05 level.

** Correlation is significant at the 0.01 level.

Table 4. Correlation matrix $\left(\mathrm{r}^{2}\right)$ between macronutrients and major constituents of essential oil

\begin{tabular}{|c|c|c|c|c|c|c|c|c|c|c|c|c|c|c|c|c|}
\hline 1 & 2 & 3 & 4 & 5 & 6 & 7 & 8 & 9 & 10 & 11 & 12 & 13 & 14 & 15 & 16 & 17 \\
\hline $\mathbf{N}(\mathbf{a v})$ & $\begin{array}{c}\mathrm{N}(\text { tota } \\
\text { l) } \%\end{array}$ & $\begin{array}{c}\mathbf{P}_{2} \mathbf{O}_{5} \\
\%(\mathbf{a} \\
\mathrm{v})\end{array}$ & $\begin{array}{c}\mathrm{K}_{2} \mathrm{O} \\
\% \\
\text { (av) }\end{array}$ & $\begin{array}{c}\text { p- } \\
\text { Cyme } \\
\text { ne }\end{array}$ & $\begin{array}{c}\gamma- \\
\text { Terpine } \\
\text { ne }\end{array}$ & $\begin{array}{c}\text { Linalo } \\
\text { ol }\end{array}$ & \begin{tabular}{|c|} 
Born \\
yl \\
acetat \\
e
\end{tabular} & $\begin{array}{c}\text { Thym } \\
\text { ol }\end{array}$ & $\begin{array}{c}\text { Carvacr } \\
\text { ol }\end{array}$ & \begin{tabular}{|c|} 
(E)- \\
Caryo \\
phylle \\
ne \\
\end{tabular} & $\begin{array}{c}\text { Germacre } \\
\text { ne D }\end{array}$ & $\begin{array}{c}\text { Bicycle } \\
\text { germacre } \\
\text { ne }\end{array}$ & $\begin{array}{c}\beta- \\
\text { Bisabole } \\
\text { ne }\end{array}$ & $\begin{array}{c}\text { Elem } \\
\text { ol }\end{array}$ & $\begin{array}{c}\text { Caryophylle } \\
\text { ne oxide }\end{array}$ & $\begin{array}{c}\text { Aliphatic } \\
\text { hydrocarbo } \\
\text { ns }\end{array}$ \\
\hline 1.00 & 0.270 & $0 . \overline{146}$ & $\begin{array}{c}- \\
0.62 \\
6\end{array}$ & -0.329 & -0.275 & $0.672^{*}$ & $0.642^{*}$ & $0.842^{* * *}$ & -0.488 & $0.684^{*}$ & $0.674^{*}$ & 0.344 & $0.649^{*}$ & 0.628 & -0.424 & -0.389 \\
\hline$b$ & 1.00 & 0.097 & $\begin{array}{c}0.26 \\
0\end{array}$ & -0.117 & -0.189 & -0.233 & -0.068 & 0.217 & -0.167 & -0.341 & -0.159 & -0.291 & -0.160 & 0.140 & 0.378 & 0.245 \\
\hline 3 & & 1.00 & $\begin{array}{c}0.36 \\
8\end{array}$ & -0.158 & -0.218 & 0.323 & -0.121 & 0.067 & 0.133 & 0.040 & -0320 & -0.068 & -0.267 & -0.381 & 0.021 & -0.143 \\
\hline & & & 1.00 & -0.158 & -0.262 & -0.488 & -0.359 & $0.709^{*}$ & -0.089 & -0.470 & -0.425 & -0.267 & -0.383 & -0.304 & $0.642^{*}$ & 0.314 \\
\hline
\end{tabular}

* Correlation is significant at the 0.05 level.

** Correlation is significant at the 0.01 level.

Table 5. Correlation matrix $\left(\mathrm{r}^{2}\right)$ among zinc $(\mathrm{Zn})$ in soil with major constituents in oil

\begin{tabular}{|c|c|c|c|c|c|c|c|c|c|c|c|c|c|c|}
\hline 1 & 2 & 3 & 4 & 5 & 6 & 7 & 8 & 9 & 10 & 11 & 12 & 13 & 14 & 15 \\
\hline $\begin{array}{c}\mathrm{Zn} \\
\text { total }\end{array}$ & $\begin{array}{c}\text { Zn } \\
\text { DTPA }\end{array}$ & $\begin{array}{c}\text { p- } \\
\text { Cymene }\end{array}$ & $\begin{array}{c}\gamma- \\
\text { Terpinene }\end{array}$ & Linalool & $\begin{array}{l}\text { Bornyl } \\
\text { acetate }\end{array}$ & Thymol & Carvacrol & $\begin{array}{c}\text { (E)- } \\
\text { Caryo } \\
\text { phyllene }\end{array}$ & $\begin{array}{c}\text { Germacrene } \\
\text { D }\end{array}$ & $\begin{array}{c}\text { Bicycle } \\
\text { germacrene }\end{array}$ & $\begin{array}{c}\beta- \\
\text { Bisabolene }\end{array}$ & Elemol & $\begin{array}{c}\text { Caryophyllene } \\
\text { oxide }\end{array}$ & $\begin{array}{c}\text { Aliphatic } \\
\text { hydrocarbons }\end{array}$ \\
\hline \begin{tabular}{|l|l|}
1 & 1.00 \\
\end{tabular} & $0.732^{*}$ & -0.122 & -0.073 & 0.075 & 0.416 & -0.335 & -0.301 & 0.173 & 0.353 & 0.012 & 0.418 & 0.516 & -0.045 & -0.177 \\
\hline \begin{tabular}{|l|l|}
2 & \\
\end{tabular} & 1.00 & -0.305 & -0.247 & 0.429 & 0.630 & -0.609 & -0.493 & 0.610 & 0.494 & 0.283 & $0.644^{*}$ & 0.410 & -0.211 & -0.341 \\
\hline 3 & & -0.136 & -0.200 & -0.097 & 0.187 & -0.197 & -0.424 & 0.033 & 0.205 & 0.019 & 0.228 & 0.337 & 0.118 & 0.059 \\
\hline
\end{tabular}

* Correlation is significant at the 0.05 level.

** Correlation is significant at the 0.01 level.

Table 6. Correlations matrix $\left(\mathrm{r}^{2}\right)$ among iron $(\mathrm{Fe})$ in soil with major constituents in oil

\begin{tabular}{|c|c|c|c|c|c|c|c|c|c|c|c|c|c|c|}
\hline 1 & 2 & 3 & 4 & 5 & 6 & 7 & 8 & 9 & 10 & 11 & 12 & 13 & 14 & 15 \\
\hline $\begin{array}{c}\mathrm{Fe} \\
\text { total }\end{array}$ & $\begin{array}{c}\text { Fe } \\
\text { DTPA }\end{array}$ & $\begin{array}{c}\text { p- } \\
\text { Cymene }\end{array}$ & $\begin{array}{c}\gamma- \\
\text { Terpinene }\end{array}$ & Linalool & $\begin{array}{l}\text { Bornyl } \\
\text { acetate }\end{array}$ & Thymol & Carvacrol & $\begin{array}{c}\text { (E)- } \\
\text { Caryo } \\
\text { phyllene }\end{array}$ & $\begin{array}{c}\text { Germacrene } \\
\text { D }\end{array}$ & $\begin{array}{c}\text { Bicycle } \\
\text { germacrene }\end{array}$ & $\begin{array}{c}\beta- \\
\text { Bisabolene }\end{array}$ & Elemol & $\begin{array}{c}\text { Caryophyllene } \\
\text { oxide }\end{array}$ & $\begin{array}{c}\text { Aliphatic } \\
\text { hydrocarbons }\end{array}$ \\
\hline \begin{tabular}{|l|l|}
1 & 1.00 \\
\end{tabular} & -0.011 & $0.693^{*}$ & $0.736^{*}$ & -0.157 & -0.034 & 0.121 & 0.449 & -0.163 & -0.223 & -0.119 & -0.094 & $\mid-0.111$ & -0.387 & -0.380 \\
\hline \begin{tabular}{|l|l|}
2 \\
\end{tabular} & 1.00 & -0.244 & -0.236 & -0.027 & 0.180 & -0.300 & -0.352 & 0.090 & $0.636^{*}$ & 0.140 & 0.382 & $0.759^{*}$ & -0.055 & -0.176 \\
\hline 3 & & 0.538 & 0.621 & -0.059 & 0.040 & -0.136 & 0.319 & 0.036 & 0.157 & 0.142 & 0.134 & 0.162 & -0.514 & -0.502 \\
\hline
\end{tabular}

$*$ Correlation is significant at the 0.05 level.

** Correlation is significant at the 0.01 level.

Table 7. Correlation matrix $\left(\mathrm{r}^{2}\right)$ among manganese $(\mathrm{Mn})$ in soil with major constituents in oil

\begin{tabular}{|c|c|c|c|c|c|c|c|c|c|c|c|c|c|c|}
\hline 1 & 2 & 3 & 4 & 5 & 6 & 7 & 8 & \begin{tabular}{|l|}
9 \\
\end{tabular} & 10 & 11 & 12 & 13 & 14 & 15 \\
\hline $\begin{array}{c}\text { Mn } \\
\text { tota } \\
1\end{array}$ & \begin{tabular}{|c|} 
Mn \\
DTP \\
A
\end{tabular} & $\begin{array}{c}\text { p- } \\
\text { Cymen } \\
\text { e }\end{array}$ & $\begin{array}{c}\gamma- \\
\text { Terpinen } \\
\text { e }\end{array}$ & $\begin{array}{c}\text { Linalo } \\
\text { ol }\end{array}$ & $\begin{array}{c}\text { Borny } \\
1 \\
\text { acetat } \\
\text { e }\end{array}$ & $\begin{array}{c}\text { Thym } \\
\text { ol }\end{array}$ & $\begin{array}{c}\text { Carvacr } \\
\text { ol }\end{array}$ & \begin{tabular}{|c|}
$(E)-$ \\
Caryo \\
phyllen \\
e \\
\end{tabular} & $\begin{array}{c}\text { Germacre } \\
\text { ne D }\end{array}$ & $\begin{array}{c}\text { Bicycle } \\
\text { germacre } \\
\text { ne }\end{array}$ & \begin{tabular}{|c|}
$\beta-$ \\
Bisabolen \\
$\mathbf{e}$
\end{tabular} & $\begin{array}{c}\text { Elemo } \\
1\end{array}$ & $\begin{array}{l}\text { Caryophylle } \\
\text { ne oxide }\end{array}$ & $\begin{array}{c}\text { Aliphatic } \\
\text { hydrocarbo } \\
\text { ns }\end{array}$ \\
\hline 11.00 & $\begin{array}{c}0.772^{*} \\
*\end{array}$ & -0.118 & -0.40 & 0.300 & 0.229 & -0.405 & -0.143 & 0.329 & 0.255 & 0.356 & 0.258 & 0,167 & -0.204 & -0.308 \\
\hline 2 & 1.00 & -0.420 & -0.381 & 0.176 & 0.216 & -0.280 & -0.446 & 0.319 & 0.418 & 0.398 & 0.343 & 0.328 & 0.056 & -0.237 \\
\hline 3 & & -0.328 & -0.258 & 0.424 & 0.618 & -0.619 & -0.598 & $0.659^{*}$ & 0.523 & 0.450 & $0.656^{*}$ & 0.392 & -0.188 & -0.330 \\
\hline
\end{tabular}

* Correlation is significant at the 0.05 level.

** Correlation is significant at the 0.01 level. 
Table 8. Correlation matrix (r) among copper $(\mathrm{Cu})$ in soil with major constituents in oil

\begin{tabular}{|c|c|c|c|c|c|c|c|c|c|c|c|c|c|c|}
\hline 1 & 2 & 4 & 5 & 6 & 7 & 8 & 9 & 10 & 11 & 12 & 13 & 14 & 15 & 16 \\
\hline $\begin{array}{c}\mathrm{Cu} \\
\text { tota } \\
\mathrm{l}\end{array}$ & $\begin{array}{c}\text { Cu } \\
\text { DTP } \\
\text { A }\end{array}$ & \begin{tabular}{|} 
p- \\
Cymen \\
e
\end{tabular} & $\begin{array}{c}\gamma- \\
\text { Terpinen } \\
\text { e }\end{array}$ & $\begin{array}{c}\text { Linalo } \\
\text { ol }\end{array}$ & $\begin{array}{c}\text { Borny } \\
\text { I } \\
\text { acetat } \\
\text { e }\end{array}$ & $\begin{array}{c}\text { Thym } \\
\text { ol }\end{array}$ & $\begin{array}{c}\text { Carvacr } \\
\text { ol }\end{array}$ & \begin{tabular}{|c|}
$(\mathrm{E})-$ \\
Caryo \\
phyllen \\
$\mathrm{e}$ \\
\end{tabular} & $\begin{array}{c}\text { Germacre } \\
\text { ne D }\end{array}$ & $\begin{array}{c}\text { Bicycle } \\
\text { germacren } \\
\text { e }\end{array}$ & $\begin{array}{c}\beta- \\
\text { Bisabolen } \\
\text { e }\end{array}$ & $\begin{array}{c}\text { Elemo } \\
1\end{array}$ & $\begin{array}{c}\text { Caryophylle } \\
\text { ne oxide }\end{array}$ & $\begin{array}{c}\text { Aliphatic } \\
\text { hydrocarbo } \\
\text { ns }\end{array}$ \\
\hline 11.00 & 0.050 & -0.236 & -0.284 & -0.061 & 0.021 & -0.232 & -0.284 & 0.047 & & 0.110 & 0.255 & 0.582 & 43 & 0.059 \\
\hline & 1.00 & \begin{tabular}{|l|}
-0.278 \\
\end{tabular} & -0.201 & 0.170 & 0.559 & -0.375 & -0.419 & 0.301 & 0.366 & 0.040 & 0.521 & 0.492 & 0.040 & -0.159 \\
\hline & & \begin{tabular}{|l|}
-0.217 \\
\end{tabular} & -0.349 & 0.049 & 0.115 & -0.204 & -0.274 & 0.087 & -0.005 & -0.176 & 0.091 & -0.023 & 0.133 & 0.300 \\
\hline
\end{tabular}

* Correlation is significant at the 0.05 level.

$* *$ Correlation is significant at the 0.01 level.

Table 9. Correlation matrix $\left(\mathrm{r}^{2}\right)$ between microclimatic conditions and major constituents of essential oil

\begin{tabular}{|c|c|c|c|c|c|c|c|c|c|c|c|c|c|c|}
\hline 1 & 2 & 3 & 4 & 5 & 6 & 7 & 8 & 9 & 10 & 11 & 12 & 13 & 14 & 15 \\
\hline $\begin{array}{c}\text { Altitud } \\
\text { e }\end{array}$ & Temp & $\begin{array}{c}\text { p- } \\
\text { Cymen } \\
\text { e }\end{array}$ & $\begin{array}{c}\gamma- \\
\text { Terpine } \\
\text { ne }\end{array}$ & $\begin{array}{c}\text { Linalo } \\
\text { ol }\end{array}$ & $\begin{array}{c}\text { Born } \\
\text { yl } \\
\text { acetat } \\
\text { e }\end{array}$ & $\begin{array}{c}\text { Thym } \\
\text { ol }\end{array}$ & $\begin{array}{c}\text { Carvacr } \\
\text { ol }\end{array}$ & \begin{tabular}{|c}
$(\mathbf{E})-$ \\
Caryo \\
phyllen \\
e
\end{tabular} & $\begin{array}{c}\text { Germacre } \\
\text { ne D }\end{array}$ & $\begin{array}{c}\text { Bicycle } \\
\text { germacre } \\
\text { ne }\end{array}$ & $\begin{array}{c}\beta- \\
\text { Bisabole } \\
\text { ne }\end{array}$ & $\begin{array}{c}\text { Elem } \\
\text { ol }\end{array}$ & $\begin{array}{c}\text { Caryophylle } \\
\text { ne oxide }\end{array}$ & $\begin{array}{c}\text { Aliphatic } \\
\text { hydrocarbo } \\
\text { ns }\end{array}$ \\
\hline 1.00 & $\mid \begin{array}{c}- \\
0.774^{*}\end{array}$ & -0.577 & -0.626 & -0.058 & 0.152 & -0.077 & -0.567 & 0.153 & 0.321 & 0.066 & 0.271 & 0.251 & 0.397 & 0.366 \\
\hline 2 & 0.108 & \begin{tabular}{|l|}
-0.194 \\
\end{tabular} & -0.336 & $-0.692^{*}$ & -0.533 & $0.706^{*}$ & -0.085 & $-0.693^{*}$ & -0.607 & -0.388 & -0.583 & -0.458 & $0.915^{* *}$ & $0.950^{* * *}$ \\
\hline 3 & \begin{tabular}{|l|}
1.00 \\
\end{tabular} & 0.393 & 0.345 & -0.286 & -0.233 & 0.238 & 0.290 & -0.379 & -0.471 & -0.252 & -0.350 & -0.319 & 0.074 & 0.168 \\
\hline 4 & & -0.087 & 0.054 & 0.516 & 0.496 & -0.623 & -0.223 & $0.644^{*}$ & $0.704^{*}$ & 0.540 & 0.627 & 0.562 & -0.583 & $-0.793^{* *}$ \\
\hline
\end{tabular}

* Correlation is significant at the 0.05 level.

** Correlation is significant at the 0.01 level.

\section{Conclusion}

The results of correlation analysis favor that essential oil composition of plants growing in wild conditions are affected by variation in soil and environmental conditions. Nitrogen and zinc in soil positively affect biosynthesis of $\beta$ bisabolene while potassium increases the amount of thymol and caryophyllene oxide and iron $\mathrm{p}$-cymene and $\boldsymbol{\gamma}$-terpinene in the collected plant material. Acidic soils, will be more favorable for the synthesis of elemol as compared to other essential oil constituents. Organically poor soil, the plant synthesized more p-cymene, $(E)$-ocimene and caryophyllene oxide.

\section{References}

1. Ashfaqullah S, Tamta S. Studies on high value medicinal plant- Origanum vulgare LA, review International Journal of Research and Analytical reviews 2018;6(1):39-44.

2. Ietswaart JH. A taxonomic revision of the genus Origanum (Labiatea), Leiden Botanical series, Lei University Press, The Hague, Leiden, Vol. 4 (1980).

3. Chishti S, Kaloo ZA, Sultan P. Medicinal importance of genus Origanum: A review Journal of Pharmacognosy and Phytotherapy 2013;5(10):70-177.

4. Chalchat JC, Pasquier B. Morphological and chemical studies of Origanum Clones: Origanum vulgare L. ssp. vulgare Journal of. Essential Oil Research 1998;10(2):119-125.

5. Vokou D, Kokkini S, Bessiere JM, Geographic variation of Greek oregano (Origanum vulgare ssp. hirtum) essential oils Biochemical Systematics and Ecology 1993;21(2):287-295.

6. Werker E. Function of essential oil-secreting glandular hair in aromatic plants of Lamiacea-A review Flavour and Fragrance Journal 1993;8(5),249-255.

7. Brdanin S, Bogdanovic N, Kolundzic M, Milenkovic M, Golic N, Kojic M, Kundakovic T. Antimicrobial activity of oregano (Origanum vulgare L.) and basil
(Ocimum basilicum L.) extract Advanced Technologies 2015;4(2):05-10.

8. Simirgiotis MJ, Felipe Parra DB, Lopez J, Munoz P, Escobar H, Parra C. Antioxidant and antibacterial capacities of Origanum vulgare L. essential oil from the arid Andean region of Chile and its chemical characterization Metabolites 2020;10:0414 (1-12).

9. Marques JD, Volcao LM, Funck GD, Kroning IS, De Silvia WP, Fiorentini AM, Rebeiro GA, Antimicrobial activity of essential oils of Origanum vulgare L. and Origanum majorana L. against staphylocoecus aureus isolated from poultry meat Industrial Crops and Products 2015;77:444-450.

10. Elshafie HS, Mancini E, Sakr S, Martino LD, Mattia CA, Feo VD et al., antifungal activity of some constituents of Origanum vulgare L. essential oil against postharvest disease of peach fruit Journal of Medicinal Food 2015;18(8):929-934.

11. Blank D, Hubner S, Alves G, Cardoso C Freitag R, Cleff M. Chemical composition and antiviral effect of extract of Origanum vulgare Advances in Bioscience and Biotecnology 2019;10(7):188-196.

12. Raveendran S, Rajadnya RK, Tilak AV, Das S, Bhalsinge R. A study to evaluate the analgesic activity of Origanum vulgare in mice using tail flick method International Journal of Basic \& Clinical Pharmacology 2019;8(10):2254-2257.

13. Han X, Parker TL. Anti-inflammatory, tissue remodeling, immunomodulatory and anticancer activity of Oregano (Origanum vulgare) essential oil in the human skin disease model Biochimie Open 2017;4:7377.

14. Khan M, Khan ST, Khan NA, Mahmood A, AlKedhairy A, Alkhathlanh ZH. The composition of the essential oil and aqueous distillate of Origanum vulgare L. growing in Saudi Arabia and evaluation of their antibacterial activity Arabian Journal of Chemistry 2018;11:1189-1200. 
15. Kilic O, Ozdemir FA. Variability of essential oil composition of Origanum vulgare L. Subsp. gracile population from Turkey Journal of essential oil bearing Plants 2016;19(8):2083-2090.

16. Kula J, Majda T, Stoyanova A, Georgiev. Chemical composition of Origanum vulgare L. essential oil from Bulgaria. Journal of essential oilbearing Plants 2007; 10(3):215-220.

17. Morshedloo MR, Salami SA, Nazeri FM, Craker L. Essential oil profile of oregano (Origanum vulgare L.) populations grown under similar soil and climate conditions Industrial Crops and Products 2018;119:183190.

18. Martino LD, Feo VD, Formisano C, Mignola E, Senatore F. Chemical composition and antimicrobial activity of the essential oil from three chemotype of Origanum vulgare L.ssp. hirtum(Link) Ietswaart growing wid in Campania(Sourthen Italy) Molecules 2009;14(8):2735-2746.

19. Sampaio BL, Batista Da Costa F. Influence of abiotic environmental factors on the main constituents of the volatile oils of Tithonia diversifolia Revista Brasilaira de Farmacognosia 2018;28(2):135-144.

20. Tucker AO, Maciarello MJ, In: (Charalambous, G Ed.) Spices, Herbs and edible fungi. Elsevier Sciences B.V., Oxford, UK 1994.

21. Kanias GD, Souleles C, Loukis A, Philotheou-Panou E. Trace elements and essential oil composition in chemotype of the aromatic plant Origanum vulgare Journal of Radioanalytical and Nuclear Chemistry 1998;227:23-29.

22. Economakis C, Skaltsa H, Demetzos C, Sokovic M, Thanos CA. Effect of phosphorus concentrationas of the nutrient solution on the volatile constituents of leaves and bract of Origanum dictamnus Journal of Agricultural and Food Chemistry 2002;50;6276-628.

23. Said-Al Ahl HAH, Omer EA, Naguib NY. Effect of water stress and nitrogen fertilizer on herb and essential oil of oregano International Agrophysics 2009;23:269275.

24. Kilmer J, Alexander L T. Method of making mechanical analysis of soils Soil Science 1949;68:1524.

25. Jackson ML, Soil Chemical Analysis. Prentice Hall Inc., New Jersey, USA. 1958, pp. 38-226.

26. Black CA, Methods of Soil Analysis, Part 1. ASA, Inc. Madison, Wisconsin, U.S.A 1965.

27. Piper CS. Soil and Plant Analysis. The University of Adelaide, Adelaide, Australia 1942.

28. Lindsay WL, Norvell WA. Development of a DTPA soil test for zinc, iron, manganese and copper. Soil Science Society of America Journal 1978;42:421-428.

29. Pande C, Tewari G, Singh S, Singh C. Chemical markers in Origanum vulgare L. from Kumaun Himalayas: a chemosystematic study Natural Product Research 2012;26(2):140-145.

30. Singh S, Tewari G, Pande C, Singh C. Chemotaxonomy of Wild Origanum vulgare L. from Kumaun Himalayas, In: (Eds. D.R. Khanna, A.K. Chopra, Gagan Matta, Vikas Singh and Rakesh Bhutiani) Impact of global climate change on earth ecosystems, Biotech Books, New Delhi, 2013, 103-114.

31. Dunford NT, Vazquez RS Effect of water stress on plant growth and thymol and carvacrol concentrations in Maxican oregano grown under controlled conditions Journal of applied Horticulture 2005;7(1):20-22.

32. Arabaci O, Bayram E, Baydar H, Savran AF, Karagodan T, Ozay N. Chemical composition, yield and contents of essential oil of Lavandula hybrid Reverchon grown under different nitrogen fertilizer, plant density and location Asian Journal of Chemistry 2007;19:2184-2192.

33. Omer, EA Response of wild Egyptian oregano to nitrogen fertilization in a sandy soil Journal of plant Nutrition 1999;22(1):103-114.

34. Srivastava NK, Misra A, Sharma S. Effect of Zn deficiency on net photosynthetic rate, ${ }_{14} \mathrm{C}$ partitioning, and oil accumulation in leaves of peppermint Photosynthetica 1997;33:71-79.

35. Marschner, H Mineral nutrient of higher plants. Second Ed., Academic Press Limited. Harcourt Brace and Company, Publishers, London, 1995, 347-364.

36. Singh S, Tewari G, Pande C, Singh C. Chemical variation in volatiles of Craniotome furcata: Correlation with soil properties Record of Natural Products 2013;7(4):332-345.

37. Singh S, Tewari G, Pande C, Singh C. Variation in essential oil composition of Ocimum ammericanum L. from north-western Himalayan region Journal of essential oil Research 2013;25(4):278-290. 